\title{
Large-Eddy Simulation of Separation Control for Compressible Flow over a Wall-Mounted Hump
}

\author{
Jennifer A. Franck* and Tim Colonius ${ }^{\dagger}$ \\ Division of Engineering and Applied Sciences, California Institute of Technology, Pasadena, CA 91125
}

\begin{abstract}
Compressible large-eddy simulations of turbulent flow over a wall-mounted hump with active flow control are performed and compared to previous experiments. We consider a range of Mach numbers from 0.1 to 0.6. Control is applied just before the natural separation point via steady suction and zero-net mass flux oscillatory forcing. Compared with the baseline flow, control shortens the separation bubble length, but is generally found to be less effective at compressible Mach numbers. The LES matches well to the available experimental data for the baseline and steady suction cases. With oscillatory forcing, the LES captures the major flow physics of the large scale shedding of vortical structures, but over-predicts the separation bubble length at low Mach numbers.
\end{abstract}

\section{Introduction}

Synthetic jets have been shown to increase aerodynamic performance of naturally separating flows in the laboratory. However, the development of accurate predictive tools for unsteady separation and control remains a challenge, especially at high Reynolds numbers. ${ }^{1}$ In order to provide the computational fluid dynamics (CFD) community with an experimental database of separated and controlled flows that can be used for computational validation, Seifert and Pack investigated the turbulent flow over a wall-mounted hump geometry. ${ }^{1-3}$ At fully turbulent Reynolds numbers the wall-mounted hump flow is characterized by its unsteady separation and the large separation bubble formed over the trailing edge. Seifert and Pack investigated many control configurations, including steady suction and oscillatory zero net-mass flux control, over a range of Mach numbers. The wall-mounted hump was also a test case at the CFD Validation of Synthetic Jets and Turbulent Separation Control workshop held at NASA Langley Research Center. ${ }^{4}$ The workshop provided a separate set of experimental data of the baseline and controlled flow including additional experimental data from pressure taps, two and three-dimensional particle image velocimetry (PIV), and oil film flow visualization along the surface of the hump. ${ }^{5,6}$ The well documented experiments from both groups provide a database that can be utilized for the development of CFD techniques capable of simulating separation and control.

Participants from the workshop simulated this flow using a variety of techniques including Reynoldsaveraged Navier-Stokes (RANS) methods and large-eddy simulation (LES). ${ }^{4}$ These methods displayed varying degrees of success in predicting the surface pressure coefficient of the baseline, steady suction, and oscillatory control test cases at a Reynolds number of $9.29 \times 10^{5}$ based on the freestream velocity $U_{\infty}$ and the chord length $c$. The method that best predicted the important flow features of separation and reattachment is the dynamic Smagorinsky LES employed by You et al. ${ }^{7}$ However this code features an incompressible flow model not capable of capturing effects of compressibility. The implicit LES (ILES) performed by Morgan et al. ${ }^{8,9}$ at a lower Reynolds number predicted the dynamics of the baseline and steady suction case, but over-predicted the separation bubble length for the oscillatory forcing likely due to the low Reynolds number. Saric et $\mathrm{al}^{10}$ found better agreement with the experiments using LES rather than RANS or detached eddy simulation (DES), but also found that the LES slightly over-predicted the reattachment point for the controlled cases.

Many of the CFD simulations used an incompressible flow model and all ran simulations to compare with the Mach 0.1 experiments from the NASA Langley workshop. Seifert and Pack have investigated

${ }^{*}$ PhD Candidate, Mechanical Engineering, Member AIAA

${ }^{\dagger}$ Professor, Mechanical Engineering, Senior Member AIAA 
the flow over a range of Mach numbers from 0.25 to 0.7 and observed the effect of compressibility on the baseline and controlled flow. ${ }^{3}$ Notably, the effect of control given the same percent of momentum or mass injection is significantly decreased at higher Mach numbers. The numerical method presented in this paper is a compressible LES capable of modeling the compressible subsonic flow over the hump. We explore the baseline flow for Mach numbers ranging from 0.1-0.6 and compare with available data from both the NASA Langley workshop experiments and those performed by Seifert and Pack. We also apply steady suction and oscillatory forcing to simulate the controlled experiments and validate our numerical control method over the same range of Mach numbers. The dynamics of the flow and the effects of compressibility are also discussed.

\section{Computational Methodology}

The governing equations are the non-density-weighted ${ }^{11}$ low pass filtered three-dimensional compressible Navier-Stokes equations given in Eq. (1).

$$
\begin{aligned}
& \frac{\partial \bar{\rho}}{\partial t}+\frac{\partial}{\partial x_{j}} \bar{\rho} \bar{u}_{j}=\frac{\partial}{\partial x_{j}} F_{m_{j}} \\
& \frac{\partial}{\partial t} \bar{\rho} \bar{u}_{i}+\frac{\partial}{\partial x_{j}}\left(\bar{\rho} \bar{u}_{i} \bar{u}_{j}-\bar{\tau}_{j i}\right)+\frac{\partial \bar{p}}{\partial x_{i}}=\frac{\partial}{\partial t} F_{m_{j}}+\frac{\partial}{\partial x_{j}} F_{u_{j} m_{j}} \\
& \frac{\partial}{\partial t} \bar{\rho} \bar{E}+\frac{\partial}{\partial x_{j}}\left((\bar{\rho} \bar{E}+\bar{p}) \bar{u}_{j}+\bar{q}_{j}-\bar{\tau}_{j i} \bar{u}_{i}\right)=\frac{\partial}{\partial t} F_{\rho E}+\frac{\partial}{\partial x_{j}} F_{(\rho E+p) u_{j}}
\end{aligned}
$$

The length scales are non-dimensionalized with the chord length, $c$, the velocities are non-dimensionalized by $a_{\infty}$ and the pressure is non-dimensionalized by $\rho a_{\infty}^{2}$. The dynamic viscosity is held constant, the Prandtl number is fixed at 0.7 , and the ideal gas law is used as the equation of state. The filtered stress tensor and heat flux vector components are given below:

$$
\begin{aligned}
& \bar{\tau}_{i j}=\mu\left(\frac{\partial \bar{u}_{i}}{\partial x_{j}}+\frac{\partial \bar{u}_{j}}{\partial x_{i}}\right)+\frac{2}{3} \frac{\partial \bar{u}_{k}}{\partial x_{k}} \delta_{i j} \\
& \bar{q}_{j}=\frac{\mu}{\operatorname{Pr}} \frac{\partial \bar{T}}{\partial x_{j}}
\end{aligned}
$$

The subgrid scale terms $F_{m_{j}}, F_{u_{j} m_{j}}$, and $F_{(\rho E+p) u_{j}}$ are modeled using the Smagorinsky formulation in Eq. (4) where $C_{\rho}, C_{s}$, and $C_{q}$ are the model coefficients that can be dynamically calculated at each grid point and at every timestep in the same manner as used by Bodony. ${ }^{12}$

$$
\begin{aligned}
& F_{m_{j}}=C_{\rho} \Delta^{2}|\bar{S}| \frac{\partial \bar{\rho}}{\partial x_{i}} \\
& F_{u_{j} m_{j}}=C_{s} \Delta^{2} \bar{\rho}|\bar{S}| \bar{S}_{i j} \\
& F_{(\rho E+p) u_{j}}=C_{q} \Delta^{2} \bar{\rho}|\bar{S}| \frac{\partial \bar{T}}{\partial x_{j}}
\end{aligned}
$$

The model coefficients can also be set constant or set to zero, which is computationally approximately $25 \%$ faster than dynamically calculating the coefficients. When the coefficients are set to zero the method will be referred to as an implicit LES (ILES). In all cases an high-order spatial filter is applied in all three coordinate directions after every full timestep to damp out grid point-to-grid point oscillations. The filter is given in Eq. (5) and is part of a class of filters developed by Visbal and Gaidonte ${ }^{13}$ and used in previous compressible LES. ${ }^{12}$ A tunable parameter of the filter, $\alpha_{f}$, is set to 0.45 in the following data. At the interior grid points, the eighth-order accurate filter is applied using a stencil size of 9 grid points, or $N=4$, with the stencil size decreased appropriately along the boundary nodes.

$$
\alpha_{f}\left(\hat{f}_{i-1}+\hat{f}_{i+1}\right)=\frac{1}{2} \sum_{n=0}^{4} a_{N}\left(f_{i+n}+f_{i-n}\right)
$$


The governing equations are solved in a computational domain with the generalized coordinates $\xi=$ $f(x, y)$ and $\eta=f(x, y)$ in the streamwise and wall normal directions. A conformal mapping from the computational domain $(\zeta=\xi+i \eta)$ to the physical domain $(z=x+i y)$ is calculated using the Schwartz-Christoffel Toolbox by Driscoll, ${ }^{14}$ capable of creating a conformal mapping from the equally spaced rectangular computational grid to an arbitrary physical grid defined by polygon vertices. The current hump geometry is defined by approximately 900 vertices denoted by the contour $\xi=0$. The smooth contour line just above the polygon boundary at $\xi=\epsilon$ is used as the lower boundary of the physical grid.

The derivatives in the computational domain are solved using a sixth-order Padé scheme in the wall normal direction with lower order schemes along the boundaries. The derivatives in the streamwise direction are computed with a forth-order optimized explicit scheme in order to easily divide the computational load for parallel computing. A Fourier spectral method is used in the spanwise direction, and the time stepping is accomplished with a forth-order Runge Kutta scheme.

\section{Simulation Details}

The geometry investigated is a wall-mounted hump that approximates the upper surface of a $20 \%$ thick Glauert-Goldschmied type airfoil. The grid and domain size are shown in figure 1 with every sixth grid point plotted. The grid points are highly clustered around the separation region and along the wall using a hyperbolic stretching function. ${ }^{15}$ The domain size is $4.9 c \times 0.909 c \times 0.2 c$, shorter than the experimental domain in the spanwise and streamwise directions in order to reduce the computational cost.

Current computations have 800 points in the streamwise direction, 160 in the wall normal direction and 64 points in the spanwise direction for a total of approximately 8.2 million points. The resolution at the point $x / c=-0.5$ on the wall is $\Delta x / c=0.0094, \Delta y / c=0.00087$, and $\Delta z / c=0.0031$ in the streamwise, wall normal and spanwise directions, respectively, and the timestep is $\Delta t=0.00035$. Baseline simulations were run for a non-dimensional time of approximately $5 \mathrm{c} / U_{\infty}$ to reach a fully developed flow, and the time averaged results were calculated over the subsequent $5-10 \mathrm{c} / U_{\infty}$.

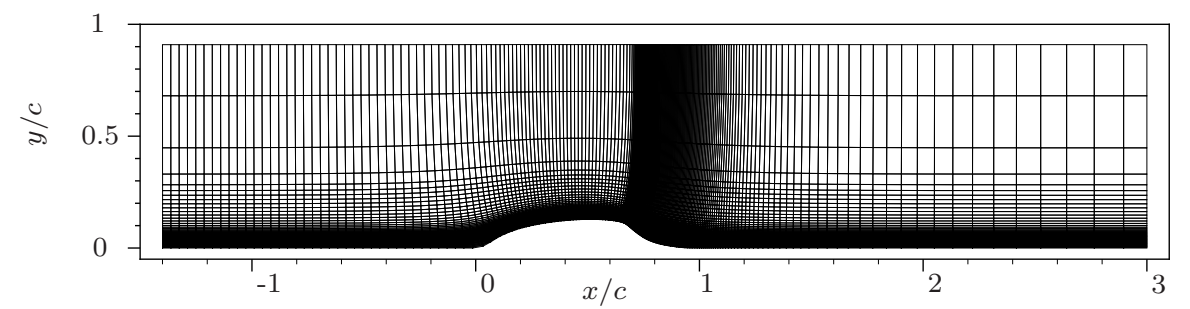

Figure 1. The computational grid (every sixth grid point plotted).

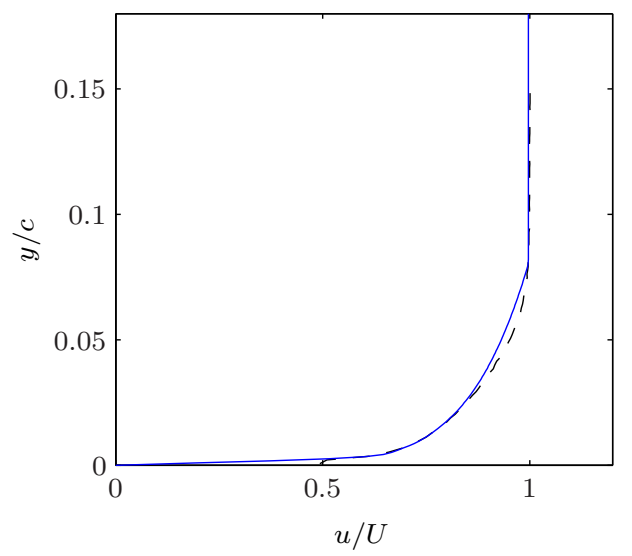

Figure 2. The inflow profile at $x / c=-1.4$ of the LES (solid line) compared with the experimental profile at $x / c=-2.14 .^{5}$ 
The flow is initialized with a potential flow solution superimposed with a turbulent boundary layer profile on the lower wall. Since the primary goal of this work is to investigate the flow separation and reattachment downstream of the hump, we do not to fully resolve the inflow turbulent boundary layer. Instead we initialize the velocity profile at the wall with velocity perturbations formulated with sums of random Fourier modes. This approach has been used in previous studies ${ }^{16,17}$ to accelerate the development of a turbulent boundary layer. The average velocity profile of the computation at the inlet location of $x / c=-1.4$ is shown in figure 2 compared with the velocity profile obtained experimentally from Greenblatt et al. ${ }^{5}$ at the upstream location $x / c=-2.14$. The boundary layer thickness in the present computations is smaller than the experiments, but it has been shown that the upstream boundary layer thickness at high Reynolds numbers has a minor effect on the flow. ${ }^{1}$

The boundary conditions are periodic in the spanwise direction, no-slip and iso-thermal conditions on the lower wall boundary, and symmetry is imposed on the upper boundary. The inflow and exit boundaries have non-reflecting boundary conditions with a buffer zone that relaxes the flow towards the initial solution. ${ }^{18}$ The Reynolds number of the simulations is 500,000 based on the chord and freestream velocity unless otherwise noted. Although the Reynolds number is lower than the test case at the NASA Langley workshop, it is within the range of Reynolds numbers investigated experimentally. ${ }^{5}$

Rather than model the flow field inside the entire actuation cavity of the experiment, the boundary conditions are modified at the wall to simulate the slot jet. ${ }^{7,19}$ When actuation is applied a normal velocity distribution is prescribed on the boundary nodes to approximate the same slot location and approximate slot width, $h_{s}$, as used in the experiments. The slot geometry and location from Greenblatt et al. ${ }^{5}$ is shown in figure 3 with the slot region enlarged on the right-hand side. Superimposed over the slot are the grid points that define the forcing width and location depicted as the positive normal velocity imposed during the blowing phase. The velocity at the wall is given by the parabolic profile ${ }^{7}$ in Eq. (6) where $0<\eta^{\prime}<h_{s}$. When steady suction actuation is applied, the fully developed baseline flow is used as the initial condition, and the negated velocity profile in Eq. (6) is gradually turned on with the ramp function given in Eq. (7). For oscillatory forcing the normal velocity at the wall is actuated in time by $\sin (\omega t)$ such that the mean slot velocity is zero.

$$
\begin{aligned}
& u_{s}=u_{s, \max } 4\left(\frac{\xi^{\prime}}{h_{s}}-\left(\frac{\xi^{\prime}}{h_{s}}\right)^{2}\right) \\
& r(t)=\frac{1}{2}\left(1+\tanh \left(3 t-\frac{1}{2}\right)\right)
\end{aligned}
$$

The steady suction controlled cases can be characterized by the mass-flux coefficient, $C_{m}$, and the steady momemtum flux coefficient, $C_{\mu}$, defined in Eq. (8), both of which are calculated using the bulk slot velocity from Eq. (6). The non-dimensional parameters for the oscillatory control are defined in Eq. (9) and are the unsteady momentum flux coefficient $\left\langle C_{\mu}\right\rangle$ based on $u_{s, \max }$ and the reduced forcing frequency $F^{+}$, in which $x_{\text {sep }}$ is $c / 2$.

$$
\begin{gathered}
C_{m}=\frac{\rho u_{s} h_{s}}{\rho U_{\infty} c} \quad, \quad C_{\mu}=\frac{\rho u_{s}^{2} h_{s}}{0.5 \rho U_{\infty}^{2} c} \\
\left\langle C_{m}\right\rangle=\frac{\rho\left\langle u_{s}\right\rangle^{2} h_{s}}{0.5 \rho U_{\infty}^{2} c} \quad, \quad F^{+}=\frac{f x_{s e p}}{U_{\infty}}
\end{gathered}
$$
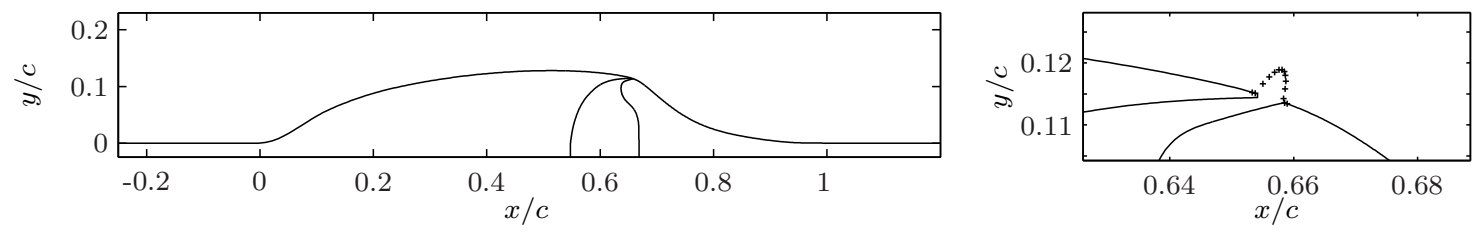

Figure 3. The hump geometry including the flow control cavity and slot used by Greenblatt et al. ${ }^{5}$ (left) and the details of the slot region compared with the forcing width and location used in the LES (right). 


\section{Results}

\section{IV.A. Baseline Flow}

The wall-mounted hump geometry represents the upper surface of a $20 \%$ thick Glauert-Goldschmied type airfoil. Two experimental groups have investigated the flow over this geometry using separate wind tunnel facilities. ${ }^{1,5}$ Figure 4 shows the surface pressure coefficient at a low Mach number from each facility. The higher suction peak obtained by Greenblatt et al. for the Langley Research Center workshop (LRCW) is likely due to the larger ratio of the model height to the wind tunnel height, creating more blockage. Another facility difference is accounted for by the endplates installed on the LRCW model. When the endplates were temporarily removed the new $C_{p}$ curve was consistently a better match to the CFD results presented at the workshop. ${ }^{5}$ However, all of the controlled cases are performed endplates still attached. To account for the increased blockage due to the endplates all experimental $C_{p}$ results from Greenblatt et al. ${ }^{5,6}$ have been rescaled by increasing the reference pressure by $0.0365 \%$. The adjusted $C_{p}$ is shown in figure 4 with the original data. Both sets of experimental data have shown that the separation and reattachment locations are relatively insensitive to Mach numbers in the range 0.1-0.25, Reynolds number above 517,000 (not shown), ${ }^{5}$ and the wind tunnel model and facility.

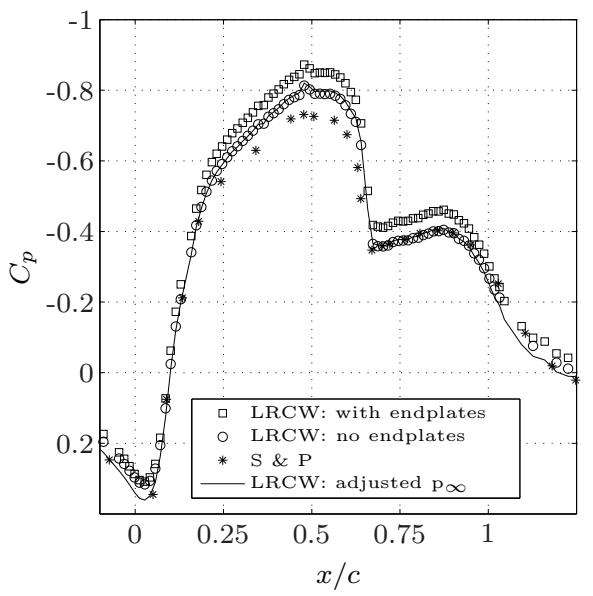

Figure 4. The facility dependence between Seifert and Pack (S\&P) and the Langley Research Center Workshop (LRCW), and the effect of endplates on the baseline flow in the LRCW facility. ${ }^{5}$

The surface pressure coefficient of the baseline flow is given in in figure 5. If the flow over the hump were fully attached, as performed by Seifert and Pack with high levels of suction control applied, ${ }^{1}$ the turbulent boundary layer upstream of the model is accelerated over the leading edge of the hump where it peaks at the highly convex region around $x / c=0.66$. A steep pressure recovery region $\left(C_{p}\right.$ increases from -1.6 to 0.5$)$ will then occur until $x / c=0.8$ when the pressure coefficient begins to level off at zero. However the natural flow separates at the highly convex region before the trailing edge $(x / c \approx 0.66)$ which hinders the pressure recovery creating a large turbulent separation bubble that reattaches at $x / c=1.1$. Thus, on an actual airfoil reattachment would not occur because there is no downstream wall to continue to the chord past $x / c=1$.

The ILES for $M=0.1$ and $\mathrm{Re}=1 \times 10^{6}$ is compared with the experimental data in figure 5 . The numerical results predict the baseline flow well, except for the slight discrepancy in the region surrounding reattachment, $0.85<x / c<1.2$. The average streamlines are plotted over the $u / U_{\infty}$ velocity contours in figure 6 and compared with the 2D PIV data ${ }^{5}$ (with endplates). Comparing the average streamline corresponding to reattachment, the ILES predicts a separation bubble approximately $9 \%$ larger than the experimental data. The mean $u / U_{\infty}$ and $v / U_{\infty}$ velocity profiles are also shown plotted against the 2D PIV data in figure 7 , and the turbulent Reynolds stresses are given in figure 8 . 


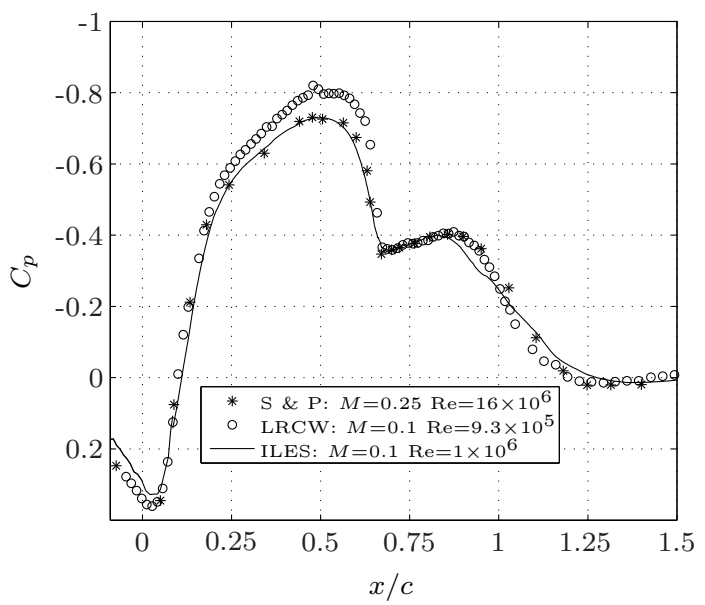

Figure 5. The ILES surface pressure coefficient compared against the experimental data.
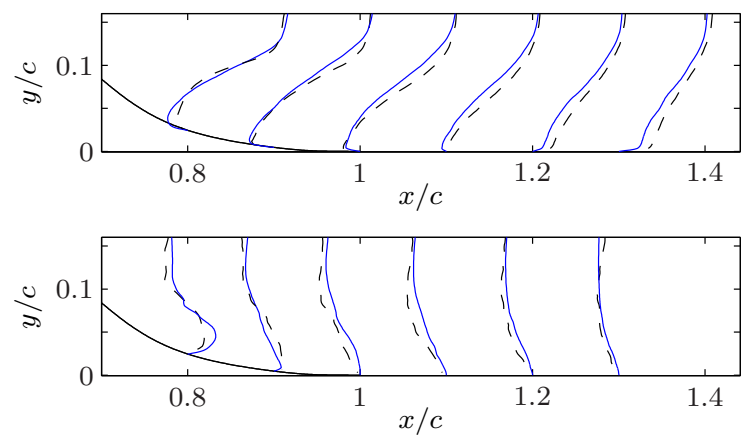

Figure 7. Baseline velocity profiles of $u / U_{\infty}$ (top) magnified by 0.8 and $v / U_{\infty}$ (bottom) magnified by 0.3 at various locations downstream of separation. Solid line is ILES and dashed line is 2D PIV data.

The ILES is also performed at higher Mach numbers and compared with available experimental data. The surface pressure coefficient is shown in figure 9 for Mach numbers of $0.1,0.3$ and 0.6. At $M=0.3$ the ILES predicts a $C_{p}$ curve very similar to the $M=0.1$ case, except that the suction peak within the separated region is not as well defined and thus lower than the ILES for $M=0.1$. As the Mach number is increased to 0.6 the flow has significantly more acceleration over the leading edge, creating a stronger low pressure region at mid-chord. The flow separates at the same physical location but has a lower pressure coefficient at separation and throughout the separated region. The most significant difference is the longer separation bubble in the compressible flow, which is captured by the ILES and also shown in experiments. ${ }^{3}$ This effect is likely due to the lower mixing rate in the compressible shear layer, which reduces the rate of fluid entrainment into the separated region and further delays the reattachment of the shear layer to the wall. The ILES for $M=0.6$ does not match the experimental results as well as the lower Mach numbers in the reattachment region, an effect that is probably due to the lower density of streamwise grid points at the reattachment location further downstream.

Large coherent structures are present in both the high and low Mach number flow, and are created by smaller structures emanating from the shear layer at separation that coalesce over the trailing edge. This is shown in the instantaneous pressure field of the mid-span plane for $M=0.1$ and $M=0.6$ shown in figure 10. The relative two-dimensional nature of these structures can be seen in the pressure isosurfaces of the $M=0.1$ 
case given by figure 11 .

The LES model was turned on for the baseline flow at Mach numbers of 0.3 and 0.6. Both a constant coefficient LES and the dynamic coefficient LES were compared against the ILES. In the dynamic LES, the constants are dynamically calculated at every location in space for each time step and averaged over the spanwise direction. To prevent numerical instabilities negative valued constants were set to zero. The pressure coefficient curves for the LES cases are given in figure 12. Due to the very small variation between the ILES and LES for the baseline case at these Mach numbers the ILES is used for the majority of the simulations presented, including cases with control.

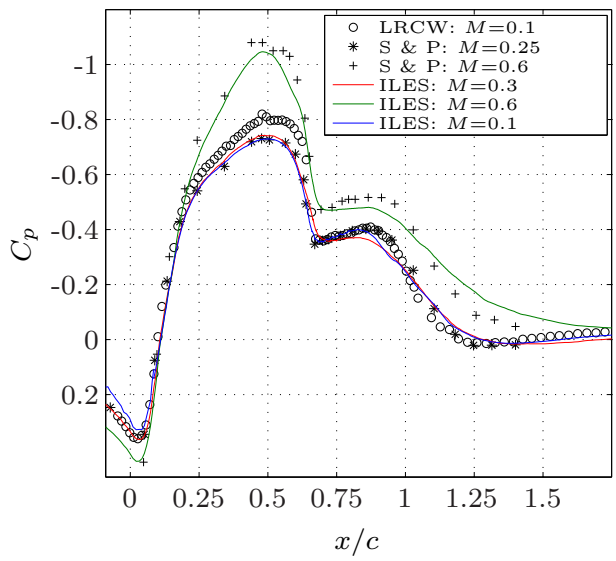

Figure 9. The surface pressure coefficient for the baseline flow for ILES at $M=0.1$ and $\mathbf{R e}=1 \times 10^{6}$, $M=0.3,0.6$ and $R e=0.5 \times 10^{6}$ compared with experimental data at $M=0.1$ and $R e=0.93 \times 10^{6}, M=0.25$ and $\mathbf{R e}=16 \times 10^{6}$, and $M=0.6$ and $\mathbf{R e}=30 \times 10^{6}$.
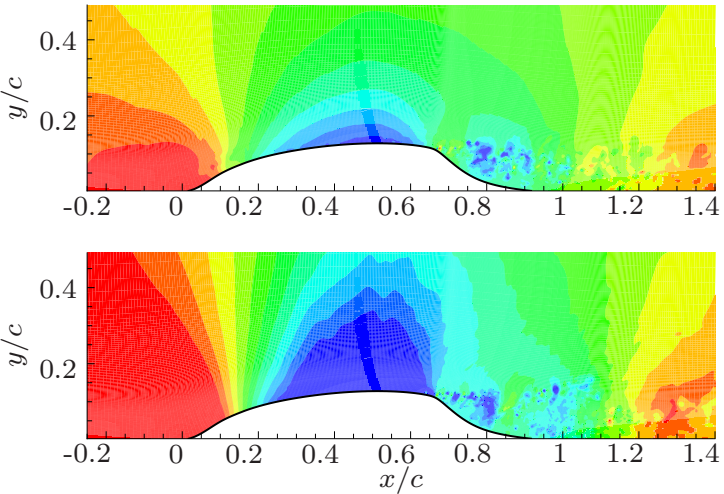

Figure 10. Instanteous pressure contours at mid-span for $M=0.1$ (top), with 11 contour levels equally spaced between 0.711 and 0.715 , and $M=0.6$ (bottom), with 11 contour levels between 0.59 and 0.715 .

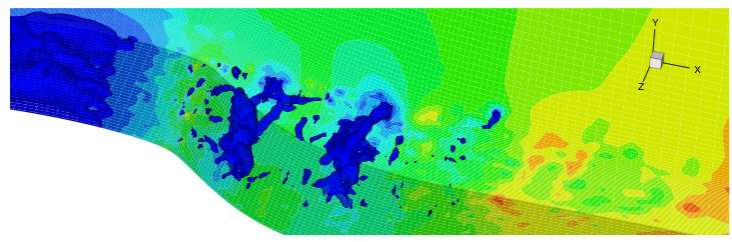

Figure 11. Instantaneous pressure contour at $M=0.1$ with same contour levels as figure 10 and pressure isosurfaces at 0.711 .

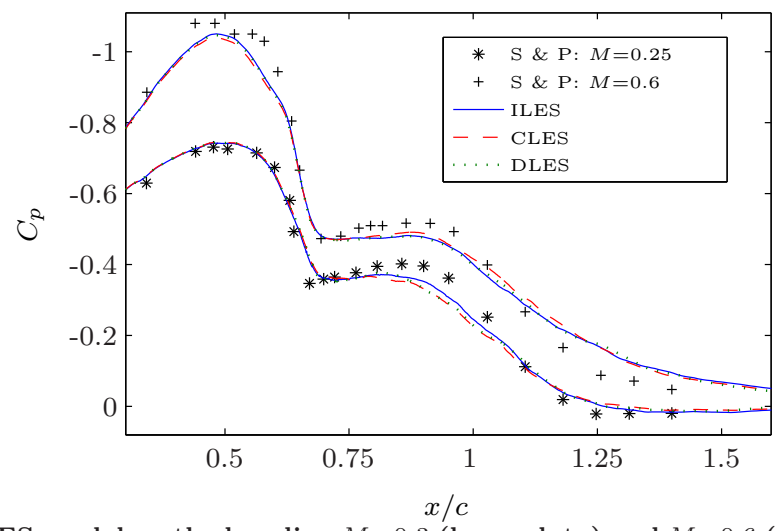

Figure 12. The effect of the LES model on the baseline $M=0.3$ (lower data) and $M=0.6$ (upper data) flows at Re= $0.5 \times 10^{6}$. In the constant coefficient LES $C_{\rho}, C_{r}$, and $C_{q}=0.06$. 


\section{IV.B. Controlled Flow}

\section{IV.B.1. Steady Suction Control}

In order to assess the ILES as a predictive tool for flow control, steady suction control is applied to the $M=0.1$ flow and compared with experimental data. The steady suction is applied just before natural separation, and has the effect of thinning the boundary layer and delaying separation. The flow therefore remains attached over the highly convex region region of the hump deflecting the shear layer downward and thus forming a smaller recirculation bubble. The effect on the surface pressure coefficient is shown in figure 14. The control creates a steep suction peak that closely resembles the attached flow, but still creates a small turbulent separated region that reattaches around $x / c=0.92$. The presence of the reduced separation bubble is also seen in the instantaneous pressure isosurface in figure 13. The structures that form in the separated region are smaller in size and not as coherent as those in the baseline case.

The pressure coefficient of the ILES at two levels of steady suction control is shown in figure 14. Due to the size of the computational forcing region, the slot width, $h_{s}$, used in the simulations is larger than the value of the slot width used to calculate the non-dimensional forcing parameters in the experiments. This discrepancy in the slot width makes it impossible to apply a level of suction that matches both the experimental value of $C_{m}$ and $C_{\mu}$ simultaneously. The dashed line in 14 corresponds to a $C_{m}$ value of $0.15 \%$, matching that reported by the workshop test case experiment, but has a $C_{\mu}$ of $0.11 \%$, lower than that in the test case experiment. The ILES captured the behavior of the flow very well, but had a slightly longer reattachment bubble length, likely due to the smaller $C_{\mu}$ value. When $C_{\mu}$ was increased to $0.24 \%$ to match the value of the experiment, the bubble length was shortened further and the ILES provided a better match to the experimental pressure coefficient. The average $u / U_{\infty}$ contours and streamlines are shown in figure 15 compared with the 2D PIV data, and show a good prediction of the separation and reattachment locations compared with the 2D PIV data. The velocity profiles and the Reynolds stress profiles in figures 16 and 17 are also an excellent match with the PIV data except for the $\overline{v^{\prime} v^{\prime}} / U_{\infty}^{2}$ profiles, which is over-predicted in the separated region.

Steady suction control was also applied to Mach numbers of 0.3 and 0.6 , shown in figure 18 . The discrepancy between the controlled $M=0.3$ and $M=0.1$ flows is greater than that of the baseline flows. For the same value of $C_{\mu}$, the $M=0.3$ case does not create as strong of a suction peak as the $M=0.1$ flow just before separation. Thus the shear layer separates at a slightly lower value of $C_{p}$, creating a longer separation bubble than that of the $M=0.1$ controlled case. This effect is more extreme with the $M=0.6$ flow, where the separation bubble is only slightly shortened with $C_{\mu}=0.09 \%$. A qualitative comparison with the $M=0.65$ experimental data in figure 19 shows a similar behavior. The decreased effectiveness of the control is also demonstrated in the spanwise vorticity plots of figure 20 . The $M=0.1$ case shows the slightly delayed separation that correlates to a deflected shear layer and smaller separated region, whereas the $M=0.6$ case only shows a slight deviation from the baseline flow.

The $M=0.6$ controlled cases are a challenge due to the high speeds encountered on the top of the hump. In experiments, Mach numbers above 0.625 develop local shock waves at the region surrounding separation. ${ }^{3}$ For the baseline case, $M=0.6$ was the highest the ILES was able to simulation because the code does not have a numerical scheme capable of shock-capturing. Figure 19 shows the pressure coefficient of the experimental data at $M=0.65$ that demonstrates the shock formation at separation. Note the higher value of the suction peak for the baseline case compared with the lower Mach numbers, and an even stronger shock that occurs with the addition of steady suction. When suction above a $C_{\mu}$ of $0.09 \%$ is applied to the ILES at $M=0.6$, a shock develops and the current LES is incapable of simulating the flow.

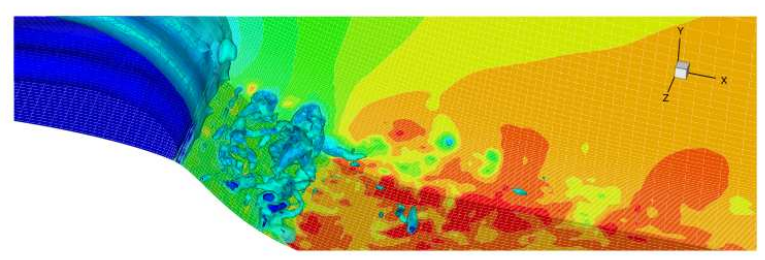

Figure 13. Instantaneous pressure contour at $M=0.1$ with steady suction with same contour levels as figure 10 and pressure isosurfaces at 0.711 and 0.712 . 


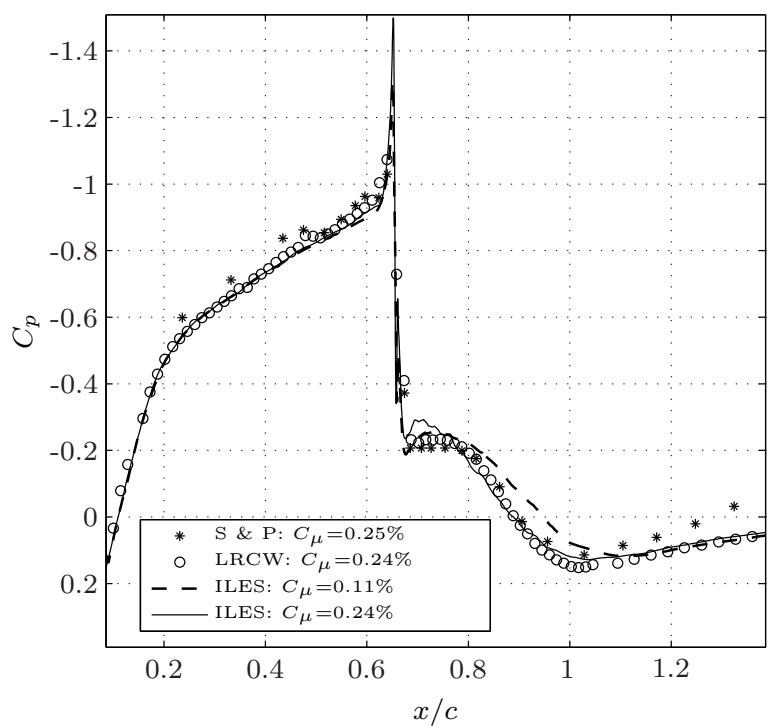

Figure 14. The ILES surface pressure coefficient for steady suction compared against the experimental data.
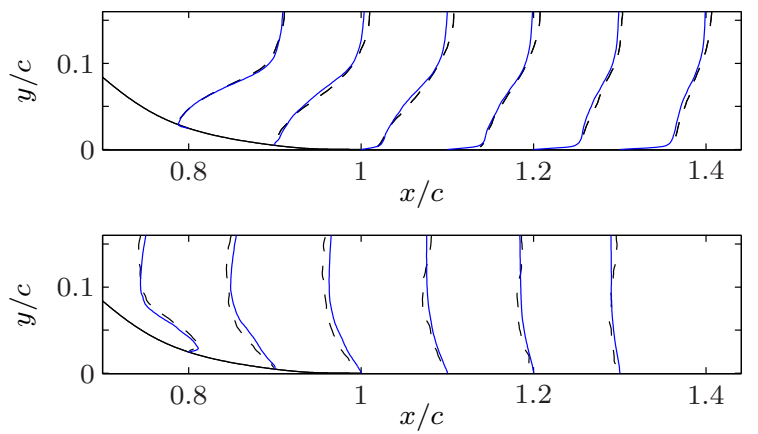

Figure 16. Steady suction velocity profiles of $u / U_{\infty}$ (top) magnified by 0.8 and $v / U_{\infty}$ (bottom) magnified by 0.3 at various locations downstream of separation. Solid line is ILES and dashed line is 2D PIV data.
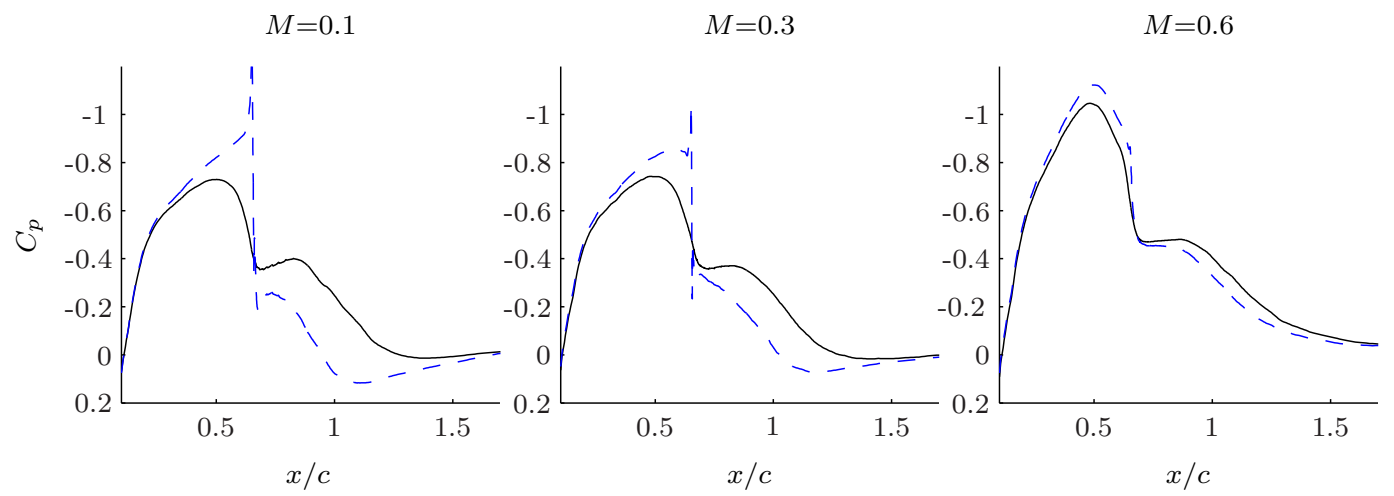

Figure 18. Baseline flow (solid line) and steady suction flow (dashed line) for $M=0.1\left(C_{\mu}=0.11 \%\right), 0.3\left(C_{\mu}=0.11 \%\right)$ and $M=0.6\left(C_{\mu}=0.09 \%\right)$. 


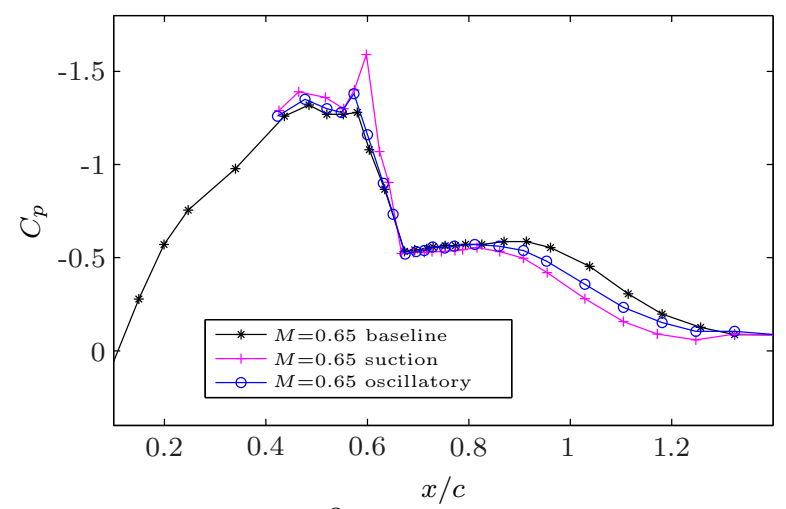

Figure 19. Experimental data from Seifert and Pack ${ }^{3}$ for $M=0.65$. Suction $C_{\mu}=.19 \%$, and oscillatory case parameters are $F^{+}=0.62$ and $\left\langle C_{\mu}\right\rangle=0.06 \%$.
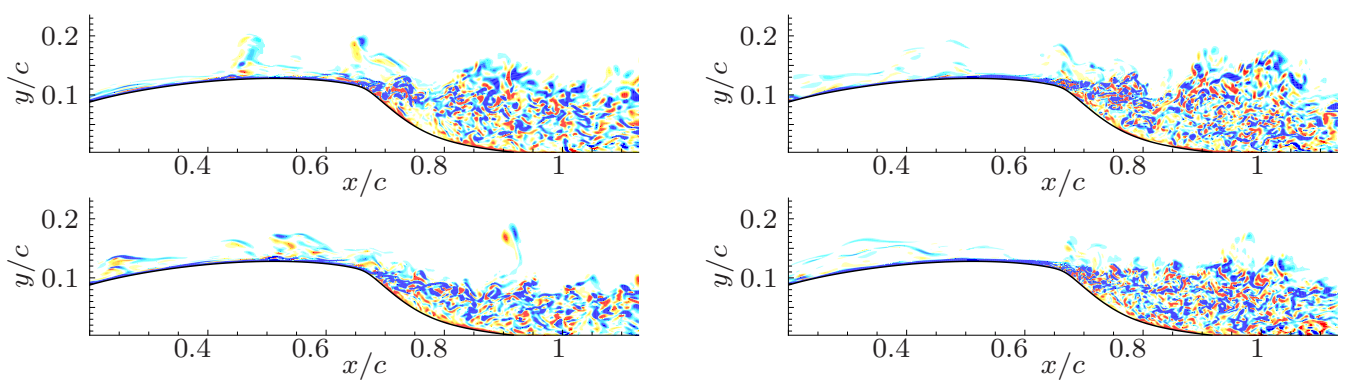

Figure 20. Baseline (top) and steady suction (bottom) instantaneous spanwise vorticity contours of the $M=0.1$ and $M=0.6$ flows. Shown are 11 contour levels from -50 to 50 .

\section{IV.B.2. Zero Net-Mass Flux Oscillatory Control}

Oscillatory forcing just before the separation point has been shown to decrease the size of the separated region, and if enough control is added, decrease the drag on the model. ${ }^{6}$ The alternating blowing and suction does not delay separation, but forms large-scale vortices that accelerate the flow's reattachment to the wall. Figure 24 gives the experimental data compared with the ILES for $M=0.1$ at $F^{+}=0.84$. The ILES does not predict the pressure coefficient for the oscillatory case as well as it does for the baseline and steady suction cases. In particular, the reattachment location for the ILES is over-predicted when compared to the experiments.

A qualitative comparison with the phase-averaged 2D PIV spanwise vorticity contours is given in 22 where phase $=90^{\circ}$ corresponds to the peak blowing and phase $=270^{\circ}$ corresponds to the peak suction. The location and size of the vortex as it is initially shed agrees well with the experiments, however it is stronger in strength than the PIV data. The shed vortex remains relatively stronger as it convected downstream and may affect the size of the separation bubble. Parameters such as the grid resolution, the LES model, and the width of the spanwise domain may improve the LES results and are currently under investigation. One incompressible dynamic LES has been able to accurately predict the $C_{p}$ curve of the oscillatory case, ${ }^{7}$ but other LES of the hump flow have also had difficulty predicting the oscillatory forced $\operatorname{case}^{9,10}$ and it has proved to be more challenging to simulate than steady suction alone.

Oscillatory forcing at $M=0.3$ was very similar to the flow dynamics at $M=0.1$, and the pressure coefficient is plotted in figure 18 against the baseline case. The $M=0.6$ case is also shown with the oscillatory forcing and baseline cases. Experimental data show a decrease in the effectiveness of oscillatory forcing at higher Mach numbers, only slightly decreasing the separation bubble length as shown in figure 19. The ILES results at Mach 0.6 show a qualitative agreement with the experimental data, however no experimental data at $M=0.6$ is available for the controlled cases. Instantaneous spanwise vorticity contours are shown in figure 24 for the oscillatory flow at $M=0.1$ and $M=0.6$. In general, the lower Mach number flow sheds vortices that are more coherent and last longer than the high Mach number oscillatory forced flow. 


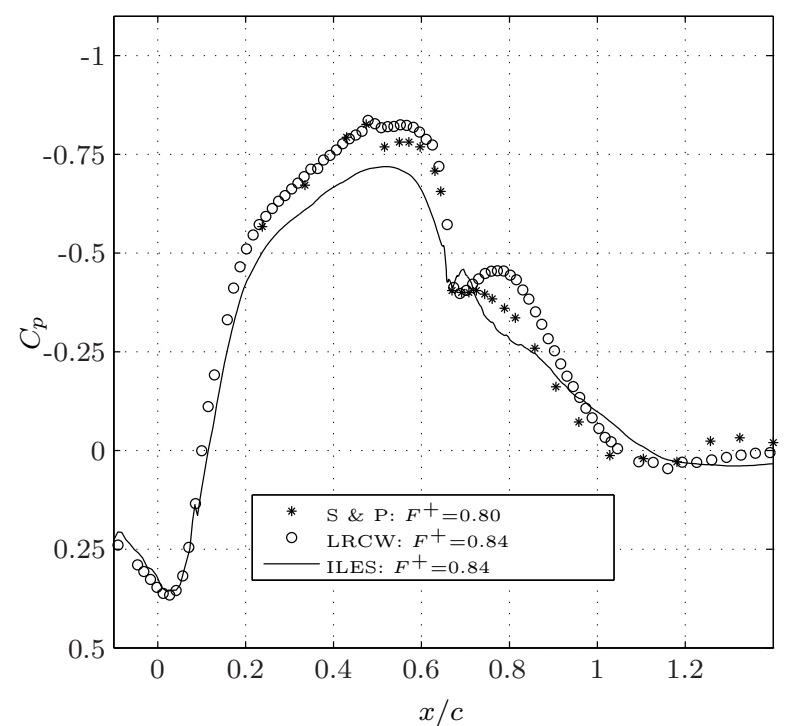

Figure 21. The ILES surface pressure coefficient for oscillatory forcing compared against the experimental data at $M=0.1$ at $\left\langle C_{\mu}\right\rangle=0.11 \%+/-0.02 \%$.
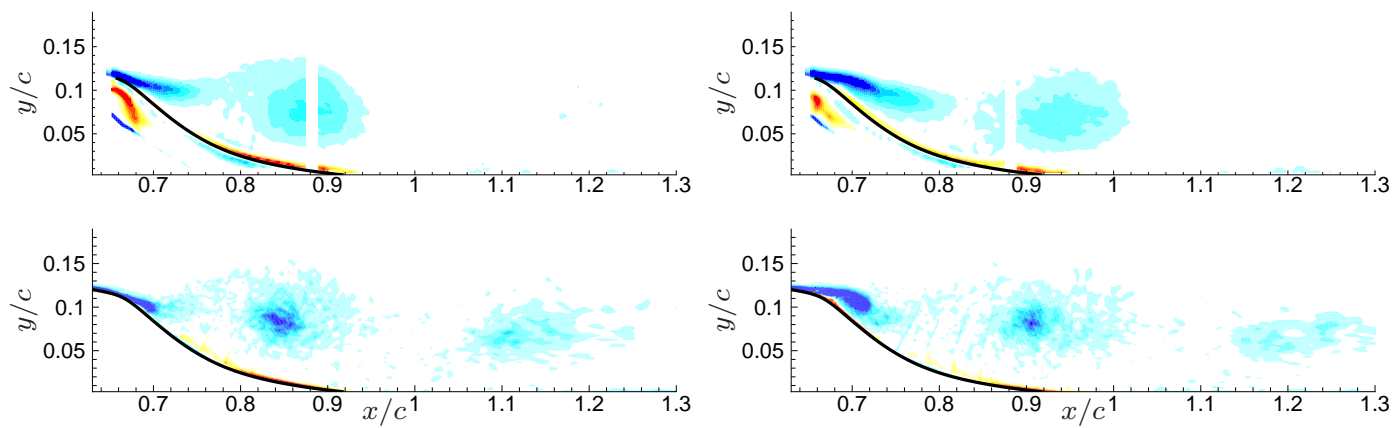

(a) phase $=0^{\circ}$

(b) phase $=90^{\circ}$
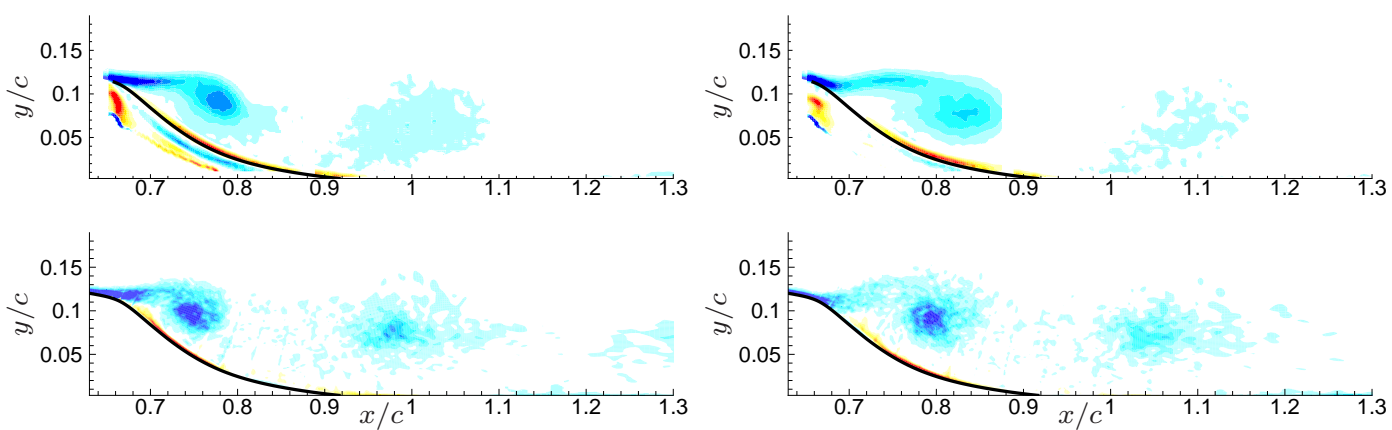

(c) phase $=180^{\circ}$

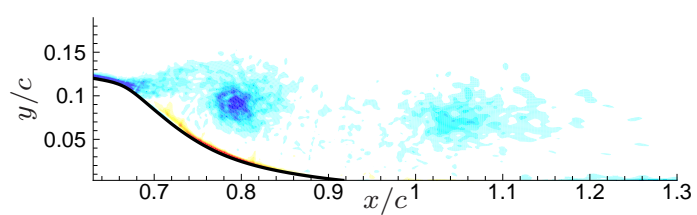

(d) phase $=270^{\circ}$

Figure 22. Phase-averaged spanwise vorticity contours of PIV data (top) and ILES (bottom) . Shown are 15 contour levels from -70 to 70 . 

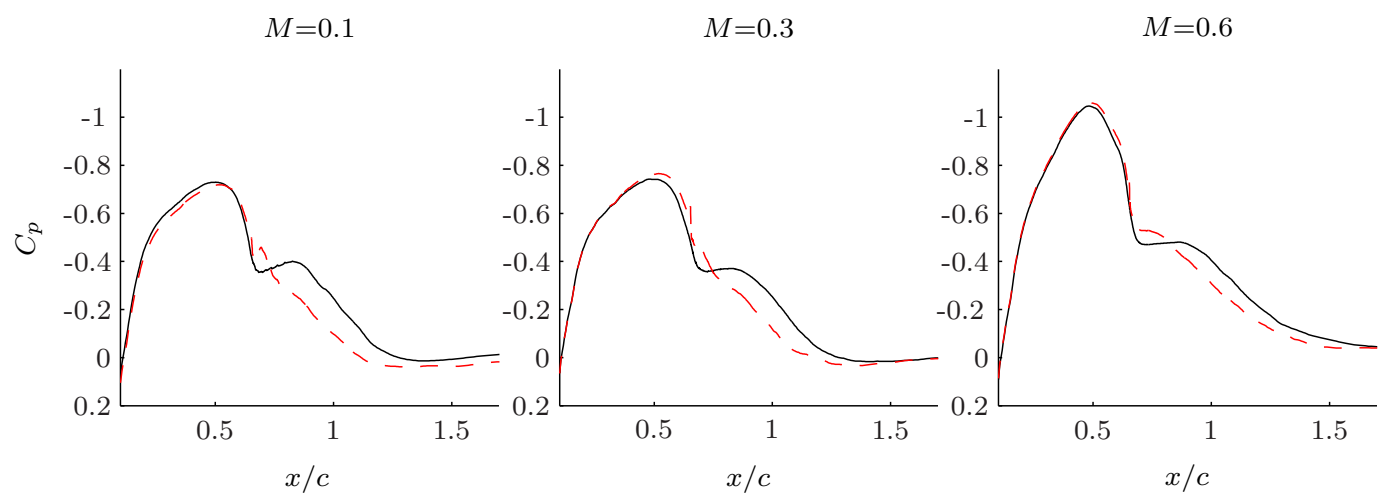

Figure 23. Baseline flow (solid line) and oscillatory forced flow (dashed line) at $F^{+}=0.84$ and $\left\langle C_{\mu}\right\rangle=0.11 \%$ for $M=0.1$, 0.3 , and 0.6 .
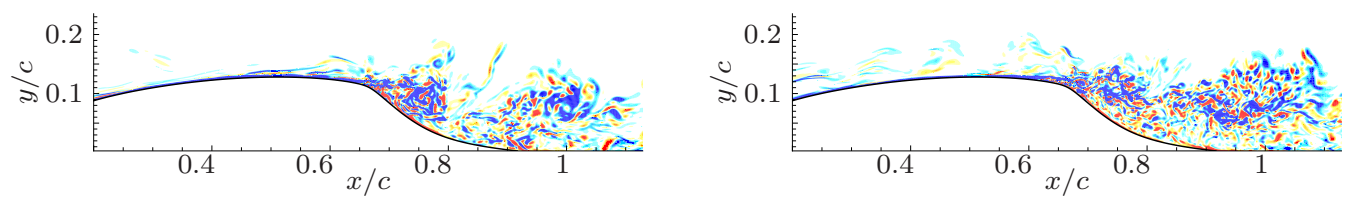

Figure 24. Instantaneous spanwise vorticity contours of the $M=0.1$ (left) and $M=0.6$ (right) oscillatory forced flows. Shown are 11 contour levels from -50 to 50 .

\section{Conclusion}

The turbulent flow over a wall-mounted hump geometry for the baseline, steady suction, and oscillatory control cases has been simulated with a compressible LES model, for a range of Mach numbers from 0.1 to 0.6. The effect of the LES model for constant coefficients and dynamically calculated coefficients was investigated for the $M=0.3$ and $M=0.6$ baseline flow cases, and it was found that the LES model does not significantly improve the simulations, thus only ILES of the controlled cases were considered.

The ILES provided a good prediction the baseline flow and steady suction control of the low Mach numbers when compared to surface pressure coefficient and 2D PIV data available from experiments. At higher Mach numbers the ILES captured the important features of the compressible flow, including the increased suction peak and longer separation bubble. The steady suction was also shown to be less effective at shortening the separation bubble at compressible Mach numbers, which is consistent with experimental results. Although the steady suction control was well predicted with the ILES, the oscillatory forcing was more difficult to accurately simulate. Using the same forcing methodology and grid resolution, the ILES over-predicted the reattachment location. However, the oscillatory forcing flow field does agree qualitatively with the available PIV results for the respective phases of the forcing. The value of the spanwise vorticity is constantly higher in the ILES results than the PIV data. In the case of oscillatory forcing, the ILES may be more sensitive to parameters such as grid resolution, domain span or the LES model coefficients, which is currently under investigation. Future work will also investigate closed-loop control algorithms to increase the effectiveness of the oscillatory forcing.

\section{Acknowledgments}

This work was partially supported by a National Science Foundation graduate student fellowship. Computational resources provided by the Department of Defense High Performance Computing Centers.

\section{References}

\footnotetext{
${ }^{1}$ Seifert, A. and Pack, L., "Active Flow Separation Control on Wall-Mounted Hump at High Reynolds Numbers," AIAA Journal, Vol. 40, No. 7, 2002, pp. 1363-1372.

${ }^{2}$ Pack, L. and Seifert, A., "Dynamics of Active Separation Control at High Reynolds Numbers," AIAA Paper 2000-0409, Jan 2000

${ }^{3}$ Seifert, A. and Pack, L., "Compressibility and Excitation Location Effects on High Reynolds Numbers Active Separation
} 
Control," Journal of Aircraft, Vol. 40, No. 1, 2003, pp. 110-119.

${ }^{4}$ Rumsey, C., Gatski, T., III, W. S., Vatsa, V., and Viken, S., "Summary of the 2004 CFD Validation Workshop on Synthetic Jets and Turbulent Separation Control," AIAA Paper 2004-2217, June 2004.

${ }^{5}$ Greenblatt, D., Paschal, K. B., Yao, C.-S., Harris, J., Schaeffler, N. W., and Washburn, A. E., "Experimental Investigation of Separation Control Part 1: Baseline and Steady Suction," AIAA Journal, Vol. 44, 2006, pp. 2820-2830.

${ }^{6}$ Greenblatt, D., Paschal, K. B., Yao, C.-S., and Harris, J., "Experimental Investigation of Separation Control Part 2: Zero Mass-Flux Oscillatory Blowing," AIAA Journal, Vol. 44, 2006, pp. 2820-2830.

${ }^{7}$ You, D., Wang, M., and Moin, P., "Large-Eddy Simuation of Flow over a Wall-Mounted Hump with Separation Control," AIAA Journal, Vol. 44, No. 11, 2006, pp. 2571-2577.

${ }^{8}$ Morgan, P., Rizzetta, D., and Visbal, M., "Large-Eddy Simulation of Flow over a Wall-Mounted Hump." AIAA Paper 2005-0484, Jan 2005.

${ }^{9}$ Morgan, P., Rizzetta, D., and Visbal, M., "Large-Eddy Simulation of Separation Flow over a Wall-Mounted Hump." AIAA Paper 2005-5017, June 2005.

${ }^{10}$ Saric, S., Jakirlic, S., Djugum, A., and Tropea, C., "Computational Analysis of Locally Forced Flow over a Wall Mounted Hump at High-Re Number"," International Journal of Heat and Fluid Flow, Vol. 27, 2006, pp. 707-720.

${ }^{11}$ Colonius, T. and Lele, S. K., "Computational aeroacoustics: progess on nonlinear problems of sound generation," Progess in Aerospace Science, Vol. 40, 2004, pp. 345-416.

${ }^{12}$ Bodony, D., Aeroacoustic Prediction of Turbulent Free Shear Flows, Ph.D. thesis, Stanford University, Dec. 2004.

${ }^{13}$ Visbal, M. and Gaitonde, D., "High-Order Accurate Methods for Complex Unsteady Subsonic Flows," AIAA Journal, Vol. 37, No. 10, 1999, pp. 1231-1239.

${ }^{14}$ Driscoll, T. A. and Trefethen, L. N., Schwarz-Christoffel Mapping, Cambridge University Press, 2002.

${ }^{15}$ Suzuki, T., Colonius, T., and Pirozzoli, S., "Vortex shedding in a two-dimensional diffuser: theory and simulation of separation control by periodic mass injection," J. Fluid Mech., Vol. 520, 2004, pp. 187-213.

${ }^{16}$ Bechara, W., Bailly, C., Lafon, P., and Candel, S., "Stochastic Approach to Noise Modeling for Free Turbulent Flows," International Journal of Aeroacoustics, Vol. 2, 2003, pp. 99-123.

${ }^{17}$ Gloerfelt, X., Bogey, C., and Bailly, C., "Numerical Evidence of Mode Switching in the Flow-Induced Oscillations by a Cavity," International Journal of Aeroacoustics, Vol. 2, No. 2, 2003, pp. 99-123.

${ }^{18}$ Freund, J. B., "Proposed inflow/outflow boundary condition for direct computation of aerodynamic sound," AIAA Journal, Vol. 35, No. 4, 1997, pp. 740-742.

${ }^{19}$ Postl, D. and Fasel, H., "Direct Numerical Simulation of Turbulent Flow Separation from a Wall-Mounted Hump," AIAA Journal, Vol. 44, No. 2, 2006, pp. 263-272. 